\title{
Potencial agronômico e divergência genética entre genótipos de berinjela nas condições do Caribe Colombiano
}

\author{
Hermes Aramendiz-Tatis ${ }^{1}$; Cláudia P Sudré²; Leandro SA Gonçalves²; Rosana Rodrigues ${ }^{2}$ \\ 'Universidad de Córdoba, Facultad de Ciências Agrícolas, Montería, Colombia, haramendiz@hotmail.com; 2UENF, Lab. de Melhora- \\ mento Genético Vegetal. Av. Alberto Lamego 2000, Pq. California, 28013-602 Campos dos Goytacazes-RJ
}

\section{RESUMO}

Foram avaliados 24 genótipos (híbridos, cultivares e variedades locais) de berinjela quanto às características produtivas no Caribe Colombiano para quantificar a divergência genética entre estes e indicar possíveis cruzamentos. Visa-se dar início a um programa de melhoramento genético da cultura na região, considerando a divergência genética e as características agronômicas desses genótipos. Utilizou-se delineamento em blocos casualizados, com três repetições. As variáveis estudadas foram comprimento do fruto, número de frutos por planta, massa média do fruto, rendimento, firmeza do fruto e altura da planta. Os dados foram submetidos à análise de variância univariada e estatísticas multivariadas utilizando-se diferentes métodos de agrupamento hierárquico e as variáveis canônicas. As médias foram agrupadas pelo teste de Scott-Knott. Verificou-se elevada divergência entre os genótipos estudados. Quando se considerou isoladamente os híbridos, as cultivares e as variedades locais, observou-se maior variabilidade entre os híbridos seguido das cultivares e, por fim, das variedades locais. O método UPGMA foi mais fidedigno no agrupamento que os métodos Ward e Vizinho Mais Próximo, pois obteve maior valor de Coeficiente de Correlação Cofenética. Pelos critérios pseudo-F e pseudo- $\mathrm{t}^{2}$ verificou-se que o ponto de máximo foi atingido quando da formação de quatro grupos, sendo este considerado o número ideal de grupos para o UPGMA. Houve concordância entre os grupos formados pelo UPGMA e pelas variáveis canônicas. Com os resultados pode-se inferir que os cruzamentos CC08 x CC02; CC08 x EU01 e CC08 x C016 têm grande potencial visando à produção e a firmeza do fruto.

Palavra-chave: Solanum melongena, análise multivariada, melhoramento de plantas, firmeza do fruto.

\begin{abstract}
Agronomic potential and genetic divergence among eggplant genotypes in the Colombian Caribbean region

We evaluated 24 genotypes (hybrids, cultivars and landraces) of eggplant related to yield traits in the Colombian Caribbean region, to quantify the genetic divergence among these genotypes and to indicate possible crosses. With the results of the genetic diversity and agronomic traits we intend to initiate a program of eggplant breeding in that region. A randomized block design with three replications was used. The analyzed variables were fruit length, number of fruits per plant, mean fruit weight, yield, fruit firmness and plant height. The data were submitted to variance analysis and the averages grouped by Scott-Knott test. A multivariate analysis was also performed using different methods of hierarchical clustering and canonical variables. There was high divergence among the studied genotypes even when considered within hybrids, cultivars and landraces, with highest variability among the cultivars and hybrids followed by the landraces. The UPGMA method was more reliable than Ward and Single Linkage methods since UPGMA presented greater value of Cophenetic Correlation Coefficient (CCC). Using pseudo-F and pseudo- $\mathrm{t}^{2}$ criteria the maximum point was reached when four clusters were formed, being considered the ideal number of groups for the UPGMA. There was agreement among the groups formed for UPGMA analysis and canonic variables. Crosses between CC08 x CC02; CC08 x EU01 and $\mathrm{CC} 08$ x $\mathrm{C} 016$ may be recommended to start an eggplant breeding program aiming to improve yield and fruit firmness.
\end{abstract}

Keywords: Solanum melongena, multivariate analysis, plant breeding, fruit firmness.

(Recebido para publicação em 10 de maio de 2010; aceito em 15 de março de 2011) (Received on May 10, 2010; accepted on March15, 2011)

\begin{abstract}
A berinjela (Solanum melongena L., $2 n=24)$ é originária das zonas tropicais da China e Índia, e foi introduzida pelos árabes na Europa, incluindo a Espanha, no período da Idade Média. Os espanhóis, por sua vez, trouxeram esta espécie para a Colômbia, onde ela se tornou parte dos hábitos alimentares do Caribe Colombiano (Aramendiz et al., 2007). Esta hortaliça é considerada de grande importância econômica e nutricional, sendo uma importante fonte de antioxidantes e minerais para a dieta humana (Rubatzky \& Yamaguchi, 1997; Kashyap et al., 2003; Kumar et al.,
\end{abstract}

2008; Raigon et al., 2008). O combate à cólera, diabetes, asma, bronquites, dor de dente e diminuição no colesterol são alguns exemplos nos quais a berinjela tem sido considerada eficaz (Daunay et al., 2001).

A Ásia é o maior produtor de berinjela, com destaques para a China e a Índia, correspondendo a 83\% da produção mundial (FAO, 2009). A Colômbia por sua vez, possui uma produção relativamente irrisória quando comparada com esses países. Entretanto, esta produção vem crescendo ao longo dos anos, principalmente na região do
Caribe Colombiano, sendo cultivada por pequenos produtores em áreas que oscilam entre 0,1 a 0,25 ha, tornando-se uma das principais hortaliças nos estados de Córdoba, Sucre e Bolívar (Aramendiz et al., 2008). Dentre os genótipos cultivados pelos agricultores encontram-se híbridos, cultivares de polinização aberta e variedades locais. Estas últimas são importantes por representarem a possibilidade de identificação de genes que podem melhorar características organolépticas e conferir resistência a fatores abióticos e bióticos em plantas (Gepts, 2006). 
Em programas de melhoramento de plantas, a informação da diversidade genética é de grande importância, pois genótipos mais divergentes, quando cruzados, originam populações segregantes com maior variabilidade e esta é uma premissa para obtenção de segregantes superiores (Hallauer \& Miranda Filho, 1986; Reif et al., 2005; Gonçalves et al., 2008).

Com o avanço da tecnologia computacional, métodos multivariados têm se tornado uma importante ferramenta para o estudo da diversidade genética (Ortiz et al., 2008). A escolha do método baseia-se na precisão desejada pelo pesquisador, na facilidade da análise e na forma como os dados foram obtidos. Entre os métodos multivariados destacam-se a análise por componentes principais, as variáveis canônicas e os métodos de agrupamento hierárquicos e de otimização (Cruz \& Carneiro, 2003; Gonçalves et al., 2008).

Os objetivos do presente trabalho foram avaliar o comportamento de oito variedades locais, sete cultivares e nove híbridos comerciais quanto às características produtivas na região do Caribe Colombiano e quantificar a divergência genética entre esses genótipos, com a perspectiva de recomendação de novos cruzamentos para geração de cultivares mais adaptadas às condições caribenhas.

\section{MATERIAL E MÉTODOS}

Vinte e quatro genótipos de berinjela do banco de germoplasma da Universidade de Córdoba e da empresa Corpoica foram avaliados (Tabela 1). Esses genótipos são oriundos da Colômbia, do Brasil, do Japão, de Taiwan e dos Estados Unidos. Deve-se ressaltar que, embora nomes comuns idênticos possam ser observados na lista dos genótipos, existem diferenças em sua genealogia, pois são produzidos por empresas diferentes (que se utilizam de diferentes genitores) e em outros casos são procedentes de diferentes locais na Colômbia, possuindo diferenças fenotípicas marcantes. Os genótipos foram semeados no primeiro semestre de 2008 no centro de pesquisa C.I. Turipaná de Cereté em Córdoba $\left(8^{\circ}\right.$ 35' $09^{\prime}$ 'N, $75^{\circ} 53^{\prime} 42^{\prime}$ 'W, altitude de 48 $\mathrm{m}$ ), com médias de umidade relativa do ar, precipitação e temperatura de $85 \%$, $1600 \mathrm{~mm}$ anuais e $28^{\circ} \mathrm{C}$, respectivamente (Palencia et al., 2006).

Os genótipos foram avaliados utilizando-se delineamento experimental em blocos completamente casualizados, com três repetições. Cada parcela foi composta por uma fileira de $10 \mathrm{~m}$ de comprimento com 11 plantas, em um espaçamento de $1 \mathrm{x} 1 \mathrm{~m}$. As mudas foram produzidas sob telado, por semeadura direta, em recipientes de polietileno de $15 \mathrm{~cm}$ de altura x $5 \mathrm{~cm}$ de diâmetro. Utilizou-se substrato composto por mistura de solo de mata com areia na proporção de 3:1. Cerca de 20 dias após a germinação, procedeu-se o desbaste das mudas, selecionando-se as mais vigorosas e deixando-se apenas uma por recipiente. As mudas foram transplantadas com $15 \mathrm{~cm}$ de altura. $O$ manejo e os tratos culturais foram feitos de acordo com as necessidades para esta hortaliça, seguindo as recomendações técnicas para a cultura conforme Aramendiz et al. (2008). Foram realizadas 13 colheitas ao longo do experimento.

As variáveis analisadas foram comprimento do fruto $(\mathrm{CF})$, em $\mathrm{cm}$; número de frutos por planta (NFP); massa média do fruto (MMF), em g; rendimento (Rend), em t/ha; firmeza (Firm) do fruto, em Newton e altura da planta (AP) $\mathrm{em} \mathrm{cm}$.

Os dados foram submetidos à análise de variância para detecção da presença de variabilidade genética entre os genótipos e, em seguida, os valores médios foram ordenados segundo o agrupamento de Scott-Knott a 1\% de probabilidade. Para a observação da variabilidade entre e dentro dos diferentes grupos de genótipos testados (híbridos, cultivares comerciais e variedades locais) utilizou-se boxplot.

Foram realizadas análises multivariadas utilizando-se técnicas de agrupamento hierárquico e de variáveis canônicas. Para a análise de agrupamento hierárquico foi utilizada a distância generalizada de Mahalanobis como medida de dissimilaridade, e no processo de agrupamento utilizaram-se os métodos UPGMA (Unweighted Pair-Group Method Using an Arithmetic Average), Ward e Vizinho Mais Próximo (VMP). Para testar a eficiência de cada método hierárquico calculou-se o coeficiente de correlação cofenética (CCC) pois, quanto maior este valor, maior é a eficiência do método em questão. Posteriormente, calculou-se o número de grupo ideal utilizando os critérios pseudo-F e pseudo- $\mathrm{t}^{2}$. Foi calculada também a importância relativa das características pelo método de Singh (1981). Os dados foram analisados pelos programas SAS (SAS Institute, 2000) e R ( R Development Core Team, 2006).

\section{RESULTADOS E DISCUSSÃO}

Pelas análises de variâncias individuais, para todos os caracteres, foram constatadas diferenças altamente significativas entre os genótipos estudados $(p \leq 0,01)$ (Tabela 2). Esse resultado indicou a necessidade de avaliação das médias separadamente em cada genótipo e sua comparação pelo teste de Scott-Knott. Houve a formação de quatro grupos para a variável NFP, cinco para Rend, seis grupos tanto para CF quanto para MMF e AP, e nove grupos para Firm (Tabela 2). Esses resultados confirmam aqueles obtidos pelo teste $\mathrm{F}$, sinalizando a existência de variabilidade genética entre os genótipos estudados. Os coeficientes de variação experimental (CVe) foram de 6,3; 6,3; 6,5; 9,2; 9,5 e 10,5 para Firm, AP, CF, NFP, MMF, Rend e NFP, respectivamente (Tabela 2). Esses valores demonstram uma alta precisão experimental dando confiabilidade aos resultados obtidos e às conclusões inferidas.

Observou-se entre as variedades locais uma menor variabilidade quando comparada com as cultivares e os híbridos para a maioria das características estudadas (Figura 1). Possivelmente, estes genótipos têm uma origem comum restringindo assim a variabilidade genética, como assinalam Prohens et al. (2005). É interessante observar no entanto, que embora tendo a menor variabilidade para o caráter $\mathrm{CF}$, as variedades locais produziram frutos de maior comprimento. A mesma observação pode ser feita para os caracteres massa média dos frutos e altura de plantas: as variedades locais tiveram menor variabilidade, porém, obtiveram os maiores valores para ambos os caracteres. Plan- 
Tabela 1. Identificação, nome comum, tipo de material e procedência dos 24 genótipos de berinjela da coleção de germoplasma da Universidade de Córdoba e da empresa Corpoica (identification, common name, material type and source of 24 genotypes of an eggplant germplasm collection of the University of Córdoba and Corpoica Company). Cordoba, Universidad de Cordoba, 2009.

\begin{tabular}{|c|c|c|c|}
\hline \multicolumn{2}{|r|}{ Genótipos } & \multirow{2}{*}{ Tipo } & \multirow{2}{*}{ Procedência } \\
\hline Código & Nome & & \\
\hline BR01 & Roxa & Cultivar & Topseed \\
\hline BR02 & Ciça & Hibrido & Embrapa \\
\hline BR03 & Linha tradicional & Cultivar & Feltrin \\
\hline $\mathrm{CC} 01$ & Long purple & Hibrido & Fercon (Colômbia) \\
\hline $\mathrm{CC} 02$ & Black Bell & Cultivar & Miguel Saen Y Cia. (Colômbia) \\
\hline $\mathrm{CC} 03$ & Barcelona & Cultivar & Impulsemillas (Colômbia) \\
\hline $\mathrm{CC} 04$ & $\mathrm{~N}^{\mathrm{o}} 5$ & Cultivar & Impulsemillas (Colômbia) \\
\hline $\mathrm{CC} 06$ & Long purple & Hibrido & Arroyave (Colômbia) \\
\hline $\mathrm{CC} 08$ & Roxa & Cultivar & Semicol (Colômbia) \\
\hline JP01 & Senryo & Hibrido & Japão \\
\hline TW01 & Chaoyan Taiwan & Hibrido & Taiwan \\
\hline TW03 & MEBH-11 & Hibrido & Taiwan \\
\hline TW04 & Mandhari seeds & Hibrido & Taiwan \\
\hline TW06 & Shuangfeeng G.13 & Hibrido & Taiwan \\
\hline EU01 & Black beauty & Cultivar & Estados Unidos \\
\hline EU02 & Long purple & Hibrido & Estados Unidos \\
\hline $\mathrm{C} 002$ & Lila & Variedade Local & Cereté, Córdoba (Colômbia) \\
\hline $\mathrm{C} 003$ & Lila pompa & Variedade Local & Cereté, Córdoba (Colômbia) \\
\hline $\mathrm{C} 009$ & Morada & Variedade Local & San Carlos, Córdoba (Colômbia) \\
\hline $\mathrm{C} 016$ & Lila & Variedade Local & Cereté, Córdoba (Colômbia) \\
\hline $\mathrm{C} 023$ & Palanca & Variedade Local & Cereté, Córdoba (Colômbia) \\
\hline $\mathrm{C} 025$ & Roja Calabaza & Variedade Local & Cereté, Córdoba (Colômbia) \\
\hline $\mathrm{C} 033$ & Morada con espina & Variedade Local & Lorica; Córdoba (Colômbia) \\
\hline $\mathrm{C} 043$ & Negra larga & Variedade Local & Montería, Córdoba (Colômbia) \\
\hline
\end{tabular}

tas altas de berinjela tendem a favorecer incidência de pragas e doenças, uma vez que plantas mais altas produzem maior densidade de copa, criando um microclima com maior umidade, que favorece o crescimento de patógenos e pragas. Entretanto, em regiões de baixa umidade a escolha por plantas altas pode ser vantajosa por estas terem um ciclo mais longo com uma maior produção de frutos por planta (Aramendiz-Tatis et al., 2008).

Já em relação aos híbridos e às cultivares, registrou-se maior variabilidade dentro de cada tipo de material genético para a maioria das características (Figura 1). Os híbridos tiveram maior variabilidade nas características CF, NFP, Rend e AP, enquanto as cultivares mostraram ampla variabilidade para MMF e Firm. As cultivares e os híbridos estudados são provenientes de regiões distintas
(Tabela 1) e são resultados de programas de melhoramento desenvolvidos em diferentes locais, certamente com objetivos variados e com a utilização de genitores diferentes fazendo com que ocorra maior variabilidade.

A cultivar $\mathrm{CC} 04$ teve o maior comprimento do fruto, com $25,4 \mathrm{~cm}$, seguida pelos híbridos JP01 e BR02 com 22,7 e $21,5 \mathrm{~cm}$, respectivamente, e pela variedade local C023 com 21,4 cm (Tabela 2). Em relação ao menor comprimento dos frutos pode-se destacar o híbrido TW03 e a cultivar CC02 com 10,6 e 12,1 cm, respectivamente. $\mathrm{O}$ tamanho do fruto está associado à demanda do mercado, ou seja, para conserva são preferidos frutos menores, como por exemplo, os acessos TW02 e CC02; entretanto, para consumo in natura adequam-se melhor os frutos entre 20 a $25 \mathrm{~cm}$ (Aramendiz-Tatis et al., 2008).
Verificou-se que o híbrido EU02 e a cultivar $\mathrm{CC} 08$ foram muito superiores em relação aos demais genótipos para a característica número de frutos por planta. Ambos os genótipos produziram 96 frutos por planta. Essa alta prolificidade se refletiu em frutos de menor massa média, com 89,0 e 84,0 g, respectivamente. Outros genótipos, como os híbridos CC06, TW03 e TW04, também revelaram alta prolificidade, com 37,7; 42,0 e 46,8 frutos por planta, e baixa massa média do fruto, com 78,0; 83,0 e 86,0 g respectivamente. Em relação às maiores massas médias dos frutos, destacaram-se o híbrido BR02, a cultivar CC02 e as variedades locais C009, C025, C043, com 315,$7 ; 340,3 ; 313,0 ; 324,0$ e 351,3 $\mathrm{g}$, respectivamente.

Os genótipos EU02, CC08, JP01 e BR02 ficaram agrupados em relação aos maiores rendimentos, com valores 
Tabela 2. Médias aritméticas ${ }^{1}$ de 24 genótipos de berinjela para seis descritores agronômicos, agrupados pelo agrupamento Scott-Knott (average values of 24 eggplant genotypes for six agronomic traits, clustered by the Scott-Knott test). Cordoba, Universidad de Cordoba, 2009.

\begin{tabular}{|c|c|c|c|c|c|c|}
\hline \multirow{3}{*}{$\begin{array}{l}\text { Genótipos } \\
\text { BR01 }\end{array}$} & \multicolumn{6}{|c|}{ Descritores agronômicos } \\
\hline & $\mathrm{CF}^{2}$ & NFP & MMF & Rend & Firm & $\mathbf{A P}$ \\
\hline & $18,0 \quad \mathrm{c}$ & $29,9 \mathrm{~d}$ & $195,3 \mathrm{~d}$ & $12,7 \quad \mathrm{c}$ & 3783,3 e & $91,1 \mathrm{c}$ \\
\hline BR02 & $21,2 \quad b$ & $29,9 \mathrm{~d}$ & 315,7 a & 20,5 a & $3916,7 \mathrm{e}$ & $102,4 \quad b$ \\
\hline BR03 & $15,7 \mathrm{~d}$ & $26,0 \mathrm{~d}$ & $290,7 \mathrm{~b}$ & $15,2 \mathrm{~b}$ & $3566,7 \mathrm{e}$ & $92,4 \mathrm{c}$ \\
\hline CC01 & $16,8 \mathrm{~d}$ & $27,3 \mathrm{~d}$ & $197,3 \mathrm{~d}$ & $7,8 \mathrm{e}$ & $3625,0 \mathrm{e}$ & $78,8 \mathrm{~d}$ \\
\hline $\mathrm{CCO2}$ & $12,1 \mathrm{f}$ & $24,7 \mathrm{~d}$ & 340,3 a & $14,0 \mathrm{c}$ & $4783,3 \mathrm{~b}$ & $75,2 \mathrm{~d}$ \\
\hline $\mathrm{CCO3}$ & $14,7 \mathrm{e}$ & $29,9 \mathrm{~d}$ & $249,3 \mathrm{c}$ & $7,3 \mathrm{e}$ & $4500,0 \mathrm{c}$ & $60,0 \mathrm{e}$ \\
\hline $\mathrm{CCO4}$ & 25,4 a & $32,1 \quad \mathrm{~d}$ & $154,3 \mathrm{e}$ & $7,2 \mathrm{e}$ & 1375,0 i & $70,5 \mathrm{~d}$ \\
\hline CC06 & $14,5 \mathrm{e}$ & $37,7 \mathrm{c}$ & $78,0 \mathrm{f}$ & $4,6 \mathrm{e}$ & $2391,7 \mathrm{~g}$ & $49,7 \mathrm{f}$ \\
\hline $\mathrm{CCO8}$ & $14,2 \mathrm{e}$ & 96,2 a & $84,0 \mathrm{f}$ & $22,2 \mathrm{a}$ & $2980,0 \mathrm{f}$ & $48,7 \mathrm{f}$ \\
\hline JP01 & $22,7 \quad b$ & $32,5 \mathrm{~d}$ & $236,7 \mathrm{c}$ & 21,1 a & $2708,3 \mathrm{~g}$ & $82,8 \mathrm{c}$ \\
\hline TW01 & $16,6 \mathrm{~d}$ & $19,5 \mathrm{~d}$ & $289,0 \mathrm{~b}$ & $11,3 \mathrm{~d}$ & $3391,7 \mathrm{e}$ & $62,2 \mathrm{e}$ \\
\hline TW03 & $10,6 \mathrm{f}$ & $42,0 \quad \mathrm{~b}$ & $83,0 \mathrm{f}$ & $8,5 \mathrm{e}$ & 7650,0 a & $49,2 \mathrm{f}$ \\
\hline TW04 & $12,7 \mathrm{f}$ & $46,8 \quad \mathrm{~b}$ & $86,0 \mathrm{f}$ & $7,0 \mathrm{e}$ & $5116,7 \mathrm{~b}$ & $55,5 \mathrm{f}$ \\
\hline TW06 & 19,9 c & $29,5 \mathrm{~d}$ & $124,0 \mathrm{e}$ & $7,4 \mathrm{e}$ & $2000,0 \mathrm{~h}$ & $63,3 \mathrm{e}$ \\
\hline EU01 & $14,2 \mathrm{e}$ & $34,7 \mathrm{c}$ & $297,3 \mathrm{~b}$ & $15,3 \mathrm{~b}$ & $5158,3 \mathrm{~b}$ & $83,2 \mathrm{c}$ \\
\hline EU02 & $14,7 \mathrm{e}$ & 96,2 a & $89,0 \mathrm{f}$ & 23,3 a & $2916,7 \mathrm{f}$ & $61,8 \mathrm{e}$ \\
\hline $\mathrm{CO02}$ & $17,6 \mathrm{c}$ & $28,2 \mathrm{~d}$ & $280,0 \quad b$ & $11,3 \mathrm{~d}$ & $3508,3 \mathrm{e}$ & 114,0 a \\
\hline $\mathrm{CO03}$ & $18,6 \mathrm{c}$ & $36,8 \mathrm{c}$ & $259,3 \mathrm{c}$ & $11,0 \quad \mathrm{~d}$ & $4141,7 \mathrm{~d}$ & $112,5 \mathrm{a}$ \\
\hline C009 & $20,1 \quad \mathrm{c}$ & $23,4 \mathrm{~d}$ & 313,0 a & $8,3 \mathrm{e}$ & $3258,3 \mathrm{f}$ & $100,3 \mathrm{~b}$ \\
\hline C016 & $18,0 \quad \mathrm{c}$ & $32,1 \quad \mathrm{~d}$ & $206,7 \mathrm{~d}$ & 13,9 c & $4608,3 \mathrm{c}$ & $98,8 \mathrm{~b}$ \\
\hline $\mathrm{CO23}$ & $21,4 \quad b$ & $30,8 \mathrm{~d}$ & $275,0 \quad b$ & $12,0 \quad \mathrm{~d}$ & $2600,0 \mathrm{~g}$ & $99,2 \mathrm{~b}$ \\
\hline $\mathrm{C025}$ & 20,4 c & $27,3 \mathrm{~d}$ & 324,0 a & $10,3 \mathrm{~d}$ & $2841,7 \mathrm{f}$ & $104,0 \quad b$ \\
\hline $\mathrm{C} 033$ & $19,0 \mathrm{c}$ & $24,7 \mathrm{~d}$ & $248,7 \mathrm{c}$ & $6,2 \mathrm{e}$ & $4091,7 \mathrm{~d}$ & $101,5 \mathrm{~b}$ \\
\hline $\mathrm{C} 043$ & $18,4 \quad \mathrm{c}$ & $24,7 \mathrm{~d}$ & 351,3 a & $9,3 \mathrm{e}$ & $2108,3 \mathrm{~h}$ & $105,3 \mathrm{~b}$ \\
\hline Média & 17,4 & 36,01 & 223,67 & 11,99 & 3625,90 & 81,77 \\
\hline QM trat ${ }^{3 /}$ & $38,51 * *$ & $36,76^{* *}$ & $25208,41 * *$ & $84,45^{* *}$ & $5165040,56^{* *}$ & $1388,49 * *$ \\
\hline $\mathrm{CVg}^{4 /}$ & 20,25 & 53,94 & 40,63 & 44,01 & 36,00 & 26,05 \\
\hline $\mathrm{Cve}^{5 /}$ & 6,49 & 10,49 & 9,25 & 9,48 & 6,29 & 6,30 \\
\hline
\end{tabular}

${ }^{1}$ Médias seguidas pela mesma letra, na mesma coluna, pertencem a um mesmo grupo (Scott-Knott); ${ }^{2} \mathrm{CF}=$ comprimento do fruto; $\mathrm{NFP}=$ número de frutos por planta; $\mathrm{MMF}=$ massa média dos frutos; Rend= rendimento; Firm= firmeza do fruto e $\mathrm{AP}=$ altura da planta; ${ }^{3} \mathrm{Quadrado}$ médio do tratamento, significativo a $1 \%$ de probabilidade; ${ }^{4}$ Coeficiente de variação genotípico; ${ }^{5}$ Coeficiente de variação ambiental $\left({ }^{1}\right.$ averages followed by the same letter in same column, belong to the same group (Scott-Knott); ${ }^{2} \mathrm{CF}=$ fruit length; $\mathrm{NFP}=$ number of fruits per plant; $\mathrm{MMF}=$ mean fruit weight; Rend= yield; Firm= fruit firmness; $\mathrm{AP}=$ plant height; ${ }^{3}$ Mean square of treatment, significant at $1 \%$ probability; ${ }^{4} \mathrm{Genotypic}$ coefficient of variation; ${ }^{5}$ Coefficient of environmental variation).

de 23,$3 ; 22,2 ; 21,1$ e $20,5 \mathrm{t} / \mathrm{ha}$, respectivamente. Ressalta-se que esse grupo foi composto por três híbridos e uma cultivar (CC08), que teve rendimento equivalente aos híbridos. Os híbridos (CC01, TW03, TW04 e TW06), as cultivares (CC03, $\mathrm{CC} 04$ e CC06) e as variedades locais (C009, C033 e C043) tiveram rendimento variando entre 4,6 a 9,3 t/ha, bem abaixo da produção média da região que é de 16 t/ha (Aramendiz et al., 2008). Esse baixo rendimento dos híbridos pode estar associado à baixa adaptação local pois, dos quatro híbridos com baixo rendimento, três são provenientes de Taiwan e apenas um da Colômbia.

Em relação à firmeza dos frutos, variável de grande importância na etapa de transporte, destacaram-se os genótipos TW03, EU01, TW04 e CC02 com valores de 7.650,0; 5.158,3; 5.116,7 e 4.783,3 Newton, respectivamente. Entretanto, os híbridos TW03 e TW04 tiveram baixos valores de rendimento, com 8,5 e $7,3 \mathrm{t} / \mathrm{ha}$, respectivamente, enquanto as variedades EU01 e CC02 tiveram valores médios de rendimento (15,3 e 14,0 t.ha ${ }^{-1}$, respectivamente). Os genótipos EU02, CC08, JP01 e BR02 que foram os mais produtivos, tiveram valores médios e baixos de resistência do fruto, com 3.916,7, 2.980,0, 2.708,3 e 3.916,7 Newton, respectivamente.

As variedades locais $\mathrm{C} 002$ e $\mathrm{C} 003$ atingiram os maiores valores para altura de planta, com 114,0 e 112,5 cm, respectivamente; enquanto a cultivar $\mathrm{CC} 08$ e os híbridos TW03 e TW04 tiveram os 


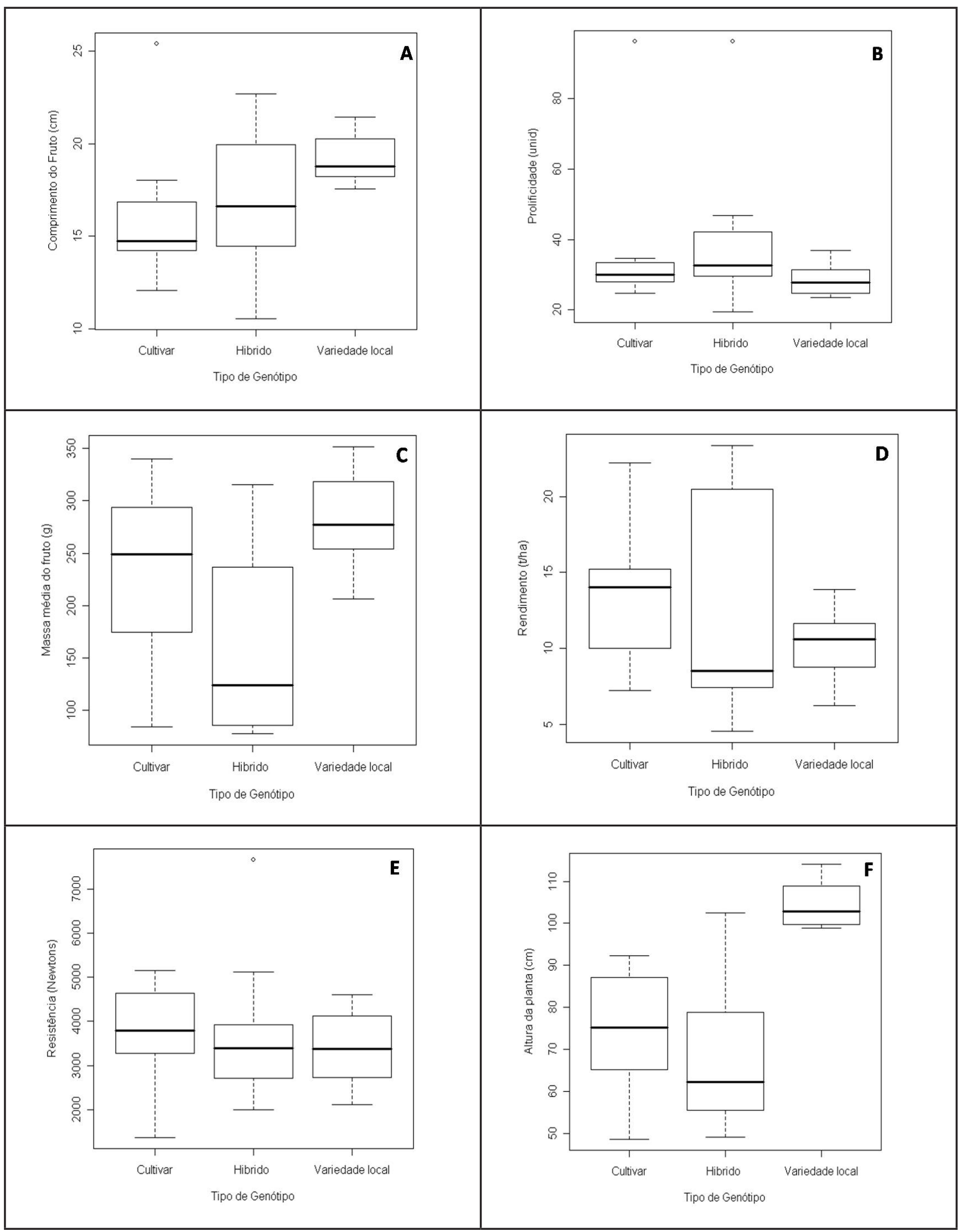

Figura 1. Boxplot dos valores mínimos, máximos e mediana - 25, 50 e 75-dos descritores comprimento do fruto (A); número de frutos por planta (B); massa média dos frutos (C); rendimento (D); firmeza do fruto (E) e altura das plantas (F) (boxplot of the minimum, maximum and median values - 25, 50 and 75 - fruit length (A); number of fruits per plant (B), mean weight of fruit (C); yield (D); fruit firmness (E) and plant height (F) traits). Cordoba, Universidade de Cordoba, 2009. 


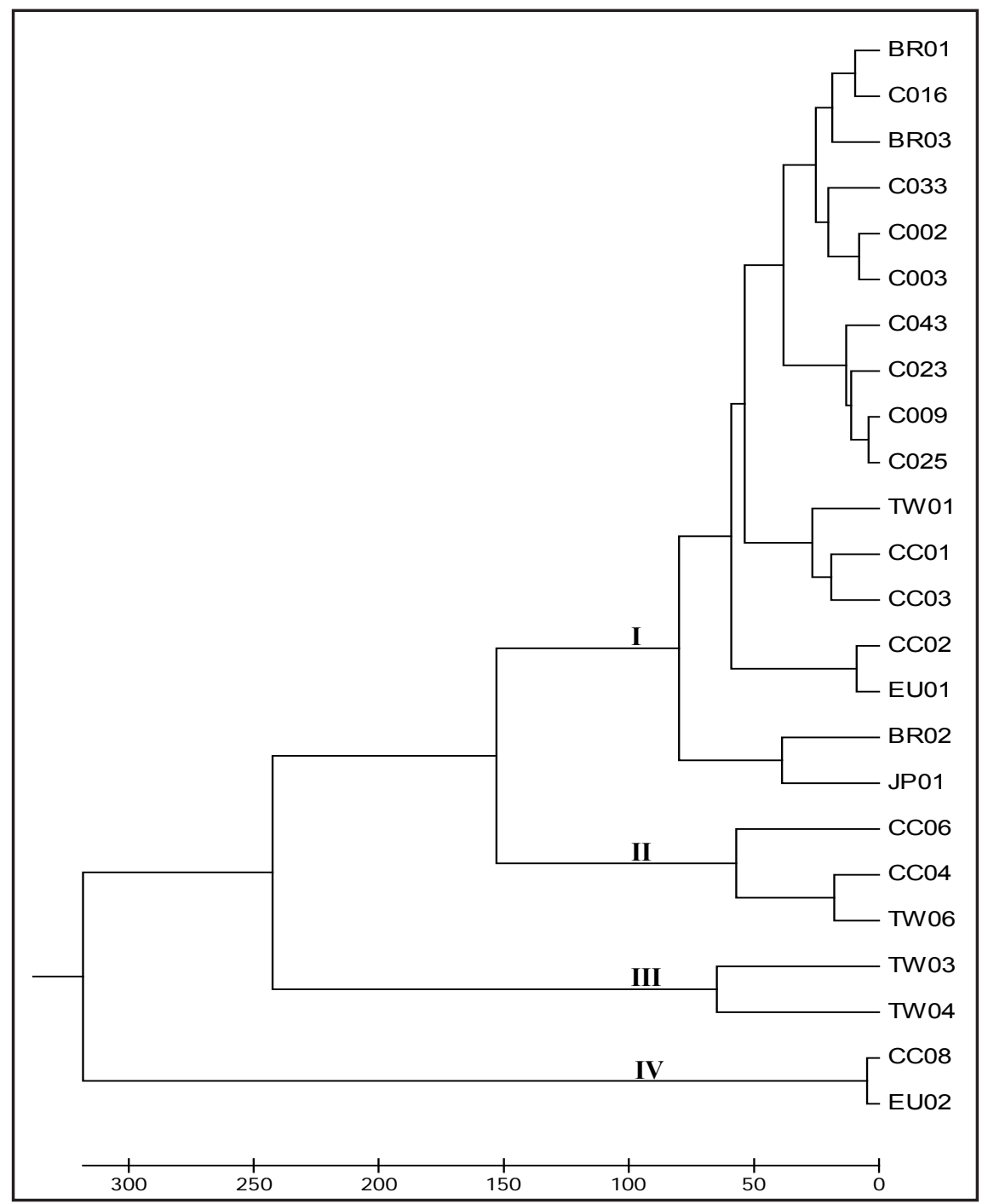

Figura 2. Dendrograma de dissimilaridades genéticas entre 24 genótipos de berinjela, obtido pelo método UPGMA com base na distância generalizada de Mahalanobis, a partir de seis descritores quantitativos (dendrogram of genetic dissimilarities among 24 eggplant genotypes, obtained by the UPGMA method based on generalized of Mahalanobis distance, from six quantitative descriptors). Cordoba, Universidade de Cordoba, 2009.

menores valores, com 48, 7; 49,2 e 55,5 $\mathrm{cm}$, respectivamente. A altura da planta influencia, além da incidência de pragas e doenças, e umidade dentro do dossel, o manejo e o ciclo da cultura. Assim, a escolha pela estatura da planta dependerá do clima da região, da mão-de-obra disponível e do tempo disponíveis para uso da área (Aramendiz-Tatis et al., 2008).

Pela análise multivariada, o método de agrupamento hierárquico UPGMA (Figura 2) mostrou ser mais fidedigno do que os métodos Ward e Vizinho Mais Próximo (VMP), uma vez que comparando os valores de correlação cofenética entre os diferentes métodos, o UPGMA teve valor de 0,9 , enquanto
Ward e VMP tiveram valores de $0,7 \mathrm{e}$ 0,8 , respectivamente. Segundo Sokal \& Rohlfe (1962), valores acima de 0,8 indicam um bom ajuste entre a matriz de similaridade ou dissimilaridade com o dendrograma. Essa maior fidedignidade do UPGMA em relação ao método de Ward e VMP, também foi encontrado por Gonçalves et al., (2008) e Rocha et al., (2009), e pode ser explicado pelo fato de que este método é baseado em médias aritméticas, enquanto o VMP considera o menor valor entre dois genótipos e o Ward considera a menor soma de quadrados em cada etapa do processo de formação dos grupos.

Pelos critérios do pseudo-F e pseu- do- $\mathrm{t}^{2}$ pode-se observar que o ponto de máximo foi atingido no passo da formação de quatro grupos, indicando que este ponto está relacionado com a menor probabilidade de significância do teste e, consequentemente indica a interrupção do processo de agrupamento. Sendo assim, quatro grupos foram determinados como ideais para análise do agrupamento UPGMA (Figura 2).

O grupo I foi constituído por 17 genótipos, sendo oito variedades locais, cinco cultivares e quatro híbridos. Este grupo revelou uma alta variabilidade para a maioria das características avaliadas. O grupo II formado pelas cultivares CC04 e CC06, e pelo híbrido TW06 teve baixos valores para firmeza dos frutos $(1.375,0 ; 2.091,7$ e $2.000,0$ Newton, respectivamente), rendimento (7,2; 4,6 e 7,4 t/ha, respectivamente) e massa média dos frutos $(154,3 ; 78,0$ e 124,0 g, respectivamente). Em relação ao grupo III, constituído pelos híbridos TW03 e TW04, os genótipos obtiveram baixos valores para comprimento do fruto $(10,6$ e $12,7 \mathrm{~cm}$, respectivamente), massa média dos frutos $(83,0$ e 86,0 g, respectivamente) e rendimento $(8,5 \mathrm{e}$ 7,0 t/ha, respectivamente). Entretanto, esse grupo teve os maiores valores para firmeza dos frutos (7.650,0 e 5.116,7 Newton, respectivamente). O grupo IV, constituído pela cultivar CC08 e pelo híbrido EU02, teve altos valores para número de frutos por planta (7,4 cada) e para rendimento $(22,3$ e $23,3 \mathrm{t} / \mathrm{ha}$, respectivamente). Contudo, os frutos deste grupo tiveram baixos valores para firmeza dos frutos $(2.980,0$ e $2.916,7$ Newton, respectivamente).

$\mathrm{Na}$ análise baseada nas variáveis canônicas (VC) verificou-se que três VC explicaram cerca de $91 \%$ da variância total, sendo $\mathrm{VC} 1$ responsável por $43,6 \%$; VC2 por $28,3 \%$ e VC3 por $19,8 \%$ adequando-se neste caso a uma representação gráfica tridimensional (Figura 3). Houve concordância entre a análise de agrupamento pelo UPGMA e as variáveis canônicas quando se observa a distribuição dos genótipos em cada grupo.

A firmeza do fruto foi a característica com maior importância relativa, ou seja, de maior contribuição para a divergência genética, com $24,8 \%$, seguido pelas 


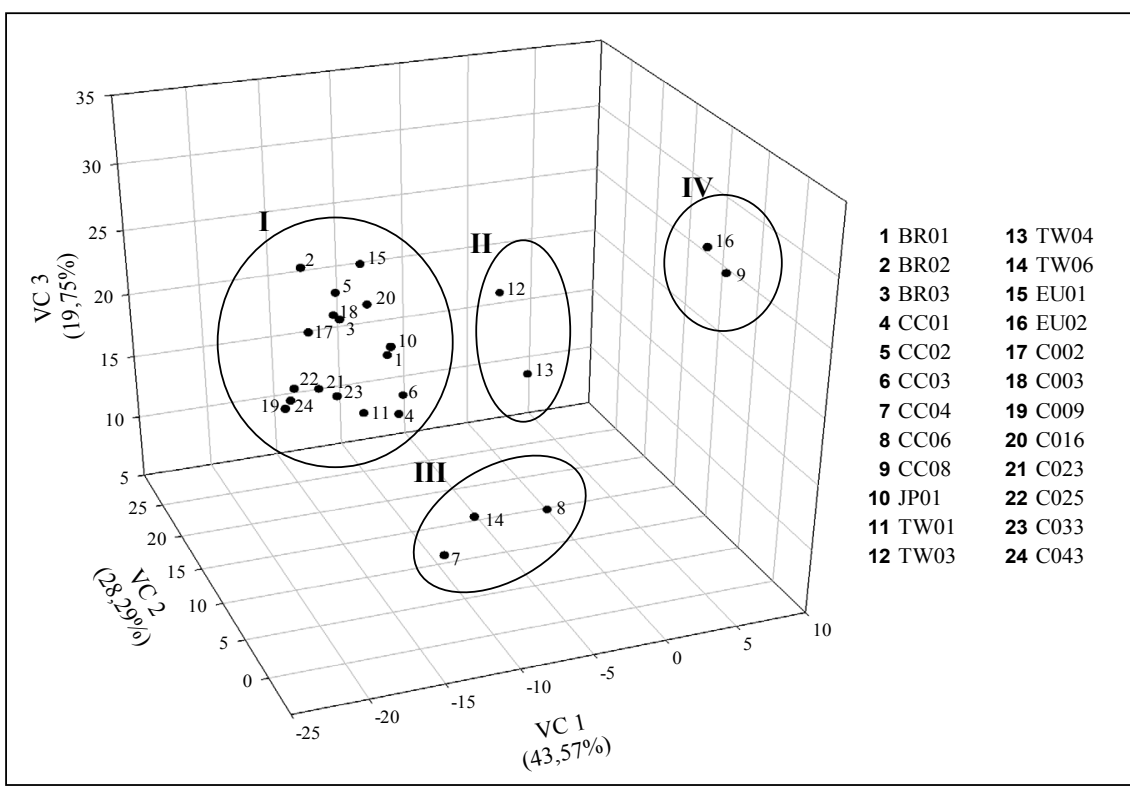

Figura 3. Dispersão gráfica dos escores em relação aos eixos representativos das variáveis canônicas (VC1, VC2 e VC3) relativos a seis descritores agronômicos em 24 genótipos de berinjela (graphic dispersion of scores on axis representing the canonical variables (VC1, VC2 and VC3) for six agronomic descriptors in 24 eggplant genotypes). Cordoba, Universidade de Cordoba, 2009.

variáveis números de frutos por planta, massa média dos frutos, rendimento e altura de planta, com 19,$9 ; 16,7 ; 15,8 ; 14,1$ e $8,7 \%$, respectivamente, demonstrando que todas as características contribuíram para a divergência genética entre os genótipos estudados.

A análise multivariada permite a predição da heterose, e alguns cruzamentos podem ser sugeridos, seguindo-se o princípio de se cruzar os genótipos mais distantes e com melhores características agronômicas. Com base nos dados obtidos neste trabalho, as seguintes combinações híbridas, a partir das cultivares mais divergentes, podem ser indicadas visando o aumento da firmeza do fruto e do rendimento: $\mathrm{CC} 08$ x CC02; CC08 x EU01 e CC08 x C016.

\section{AGRADECIMENTOS}

Ao Ministerio de Agricultura y Desarrollo Rural, Asohofrucol e à Universidad de Córdoba pelo financiamento da pesquisa.

\section{REFERÊNCIAS}

A; ROBLES J; MONTALVÁN R. 2007. Efectos del almacenamiento en la calidad fisiológica de la semilla de berenjena (Solanum melongena L.). Agronomia Colombiana 25: 104-112.

ARAMENDIZ-TATIS H; CARDONA C; JARMA A; ESPITIA M. 2008. El cultivo de la berenjena (Solanum melongena L.). Córdoba: Editorial Produmedios. 151p.

CRUZ CD; CARNEIRO PCS. 2003. Modelos biométricos aplicados ao melhoramento genético. Viçosa: Imprensa Universitária. $585 \mathrm{p}$.

DAUNAY MC; LESTER RN; GEBHARDT C; HENNART JW; JAHN M; FRARY A; DOGANLAR S. 2001. Genetic resources of eggplant (Solanum melongena) and allied species: a new challenge for molecular geneticists and eggplant. In: VAN DEN BERG RG; BARENDSE GWM; VAN DER WEERDEN GM; MARIANI C (eds). Solanaceae V: Advances in Taxonomy and Utilization. Nijmegen: Nijmegen University. p. 251-274.

FAO - Food and Agriculture Organization of the United Nations. 2009, 14 de Janeiro. Disponível em http://www.faostat.fao.org.

GEPTS P. 2006. Plant genetic resources conservation and utilization: the accomplishments and future of a societal insurance policy. Crop Science 46: 2278-2296.

GONÇALVES LSA; RODRIGUES R; AMARAL JÚNIOR AT; KARASAWA M; SUDRÉ CP. 2008 Comparison of multivariate statistical algorithms to cluster tomato heirloom accessions. Genetics and Molecular Research 7: 1289-1297.
HALLAUER AR; MIRANDA FILHO JB. 1986. Quantitative genetics in maize breeding. Ames: Iowa State University Press. 468p.

KASHYAP V; VINOD KUMAR $\mathrm{S}$; COLLONNIER C; FUSARI F; HAICOUR R; ROTINO GL; SIHACHAKR D; RAJAM MV. 2003. Biotechnology of eggplant. Scientia Horticulturae 97: 1-25.

KUMAR G; MEENA BL; KAR R; TIWARI SK; GANGOPADHYAY KK; BISHT IS; MAHAJAN RK 2008. Morphological diversity in brinjal (Solanum melongena L.) germplasm accessions. Plant Genetic Resources 6: 232-236.

ORTIZ R; CROSSA J; FRANCO J; SEVILLA R; BURGUEÑO J. 2008. Classification of Peruvian highland maize races using plant traits. Genetics Resources and Crop Evolution 55: 151-162.

PALENCIA G; MERCADO T; COMBATT E. 2006. Estudio agroclimático del departamento de Córdoba. Montería: Gráficas del Caribe Ltda. 128p.

PROHENS J; BLANCA J; NUEZ F. 2005. Morphological and molecular variation in a collection of eggplants from a secondary center of diversity: Implications for conservation and breeding. Journal of the American Society for Horticultural Science 130: 54-63.

R DEVELOPMENT CORE TEAM. 2006. A language and environment for statistical computing. Vienna: R Foundation for Statistical Computing. Capturado em 13 de março de 2008. Disponível em http://www.rproject.org/

RAIGÓN MD; PROHENS J; MUÑOZ-FALCÓN JE; NUEZ F. 2008. Comparison of eggplant landraces and commercial varieties for fruit content of phenolics, minerals, dry matter and protein. Journal of Food Composition and Analysis 21: 370-376.

REIF JC; MELCHONGER AE; FRISH M. 2005. Genetical and mathematical properties of similarity and dissimilarity coefficients applied in plant breeding and seed bank management. Crop Science 45: 1-7.

ROCHA MC; GONÇALVES LSA; CORRÊA FM; RODRIGUES R; SILVA SL; ABBOUD ACS; CARMO MGF. 2009. Descritores quantitativos na determinação da divergência genética entre acessos de tomateiro do grupo cereja. Ciência Rural 39: 664-670.

RUBATZKY VE; YAMAGUCHI M. 1997. World vegetables: principles, production and nutritive values. New York: Chapman \& Hall. 843p.

SAS INSTITUTE Inc. 2000. SAS/STAT, Version 8.2. Cary: NC.

SINGH D. 1981. The relative importance of characters affecting genetic divergence. Indian Journal of Genetic and Plant Breeding 41: 237-245.

SOKAL RR; ROHLF FJ. 1962. The comparison of dendrograms by objective methods. Taxon 11: 33-40. 